\title{
Increasing Awareness About Male Infertility: An Overview of the Sperm DNA Fragmentation Study Group (SFRAG) Guidelines
}

\author{
Sandro C. Esteves ${ }^{凶}$ \\ Sandro C. Esteves: https://orcid.org/0000-0002-1313-9680 \\ ANDROFERT, Andrology and Human Reproduction Clinic, Referral Center for Male Reproduction, Campinas, Brazil \\ Department of Surgery (Division of Urology), University of Campinas (UNICAMP), Campinas, Brazil. Faculty of Health, Aarhus University, Aarhus, Denmark
}

Sperm DNA integrity is indispensable for the birth of healthy offspring[1]. Increasing evidence indicates that sperm DNA fragmentation (SDF), a marker of damaged chromatin, has an independent and critical role in male infertility and reproductive success[2]. The reasons relate to the often higher SDF levels in ejaculated semen of infertile men (versus their fertile counterparts) and the adverse impact of SDF on the sperm's ability to fertilize the egg and promote healthy embryo development $[2,3]$. Consequently, couples in whom the male partner exhibits high SDF on neat semen may have longer time-to-pregnancy, increased risk of pregnancy loss, and decreased success in medically assisted reproductive (MAR) modalities (eg, intrauterine insemination [IUI] and in vitro fertilization/intracytoplasmic sperm injection [IVF/ICSI])[4]. The adverse effect of SDF on reproductive success is modulated by the oocyte's DNA repair capacity intrinsically related to female age[5]. Sperm DNA damage exceeding the oocyte's repair capacity-or the oocyte's failure to repair DNA damage-negatively influences the embryo's development potential and the offspring's health[6].

Routine semen analysis-the laboratory backbone of infertility investigation-has shown little progress over the last century. Semen analysis results have low discriminatory power (except at extremely low levels) as there is considerable overlap between semen characteristics (eg, sperm count, motility, and morphology) of fertile and infertile men[7]. The need for more robust male infertility diagnosis methods has been the driving force of the ongoing efforts to develop and implement SDF testing in clinical practice. Indeed, SDF tests represent one of the best examples of translational medicine in andrology.

While it is not a replacement for the current tools for infertility diagnosis, SDF testing may add independent information about sperm quality at the molecular level, and its integration into practice may provide better counseling, diagnosis, and treatment planning. Despite that, societies like the American Urological Association and the American Society for Reproductive Medicine have not recommended using SDF testing routinely during the infertility evaluation, albeit acknowledging that the tests' results might be informative. Insufficient clinical data, tests' technical limitations and lack of effective treatment options to overcome SDF-related infertility have been the common grounds for the reluctance to endorse the clinical application of SDF tests[8]. However, evidence on these areas has increased steadily, justifying the development of clinical practice guidelines to refine efficiency in diagnosing and treating clinical conditions associated with SDF.

\section{Key Words}

Male infertility, practice guideline, sperm DNA fragmentation, semen analysis, assisted reproductive technology

\section{Competing Interests}

SCE declares the receipt of unrestricted research grants and lecture fees from Merck outside the submitted work. The author is a member of the Sperm DNA Fragmentation Group and lead author of the SFRAG guidelines.

\section{Article Information}

Received on November 2, 2020

Accepted on December 13, 2020

Soc Int Urol J.2021;2(2):129-132

DOI: https://doi.10.48083/WNAU8209 
This commentary highlights the recently published evidence-based guideline from the Sperm DNA Fragmentation Study Group (SFRAG), which provides a comprehensive summary about the role of SDF in infertility and offers best practice advice on testing and care of couples with SDF[9]. The primary goals of the SFRAG guideline are to provide cliniciansurologists, andrologists, gynecologists, and reproductive endocrinologists-with clear advice on best practices in SDF. The SFRAG recommendations were based on the best available evidence, which ranged from moderate to low quality. The guideline may be used to help standardize care while securing physician autonomy, making it an invaluable resource for a broad range of professionals providing infertility care, particularly urologists.

The first part of the SFRAG guideline outlines the SDF pathophysiology and explains each SDF test. In brief, many conditions, including varicocele, chronic illness, accessory gland infections, advanced paternal age, lifestyle, obesity, occupational and environmental factors, medications, ionizing and non-ionizing radiation, and heat exposure, have been associated with elevated SDF levels. These conditions can promote SDF by causing defective spermatogenesis, evoking abortive apoptosis, or increasing the generation of reactive oxygen species (ROS). Excessive ROS causes oxidative stress, a significant causative factor of SDF in live sperm. The SFRAG guideline states there are 4 reliable tests to measure SDF. These tests are grouped in methods that use (a) enzymatic reactions to label the DNA breaks (eg, terminal deoxynucleotidyl transferase-mediated dUTP-biotin nick end labeling [TUNEL] assay) and (b) controlled DNA denaturation combined with protein depletion as intermediates to reveal the DNA breaks (eg, sperm chromatin structure assay [SCSA], sperm chromatin dispersion test [SCD], and the Comet assay). This guideline includes 13 recommendations on how testing should be carried out and results analyzed. Notably, it states that a standardized protocol with strict quality control is essential for a reliable test result and that a neat semen sample should be used for SDF testing, collected after ejaculatory abstinence of 2 to 5 days. It notes that although the results provided by the most common SDF tests do not necessarily line up, there is a good correlation between SDF rates reported by TUNEL, SCSA, SCD, and alkaline Comet. Thresholds of about $20 \%$ by TUNEL, SCSA, SCD, and alkaline Comet, assessed on neat semen, best discriminate fertile from infertile men. Additionally, thresholds of $20 \%$ to $30 \%$ evaluated by SCSA, alkaline Comet, and SCD are clinically useful for classifying infertile couples into those with a statistical probability of longer time to achieve natural pregnancy, a decreased chance of pregnancy with MAR, and an increased miscarriage risk. Lastly, it highlights that a fixed ejaculatory abstinence length should be used for SDF testing when monitoring the effects of medical and surgical interventions aimed at decreasing SDF levels.

The second part describes 7 clinical situations that may benefit from SDF testing:

1. Varicocele

2. Unexplained/idiopathic infertility

3. Recurrent pregnancy loss

4. Intrauterine insemination

5. In vitro fertilization/intracytoplasmic sperm injection

6. Infertility risk factors

7. Sperm cryopreservation.

- Varicocele: Repairing a clinical varicocele may alleviate SDF, potentially increasing the likelihood of reproductive success. SDF testing may help identify patients with a profile that would not fit the standard indication of varicocele repair (eg, clinical varicocele of any grade and normal/borderline routine semen analysis) but would benefit from varicocele repair. SDF testing may be used to monitor treatment outcomes. However, SDF testing in subfertile men with subclinical varicocele is not currently recommended.

- Unexplained/idiopathic infertility: Couples with unexplained/idiopathic male infertility should be informed that abnormal SDF levels may adversely impact their chances of achieving a live birth. An abnormal test result should prompt a complete male fertility evaluation by a reproductive urologist to help identify and possibly treat conditions associated with poor sperm DNA quality. ICSI may be considered if no correctable male factor is identified, or if abnormal SDF levels persist after treatment, particularly among couples with a limited reproductive time window.

- Medically assisted reproduction: Infertile couples eligible for MAR treatment should be informed that abnormal SDF levels may adversely impact their chances of achieving a live birth. As in idiopathic/ unexplained infertility, a reproductive urologist's evaluation is recommended to help identify and possibly treat conditions associated with SDF. Among couples with ICSI failure and elevated SDF, sperm retrieved from the testis may be considered for sperm injection in subsequent treatment cycles because of the lower SDF rates in testicular than in epididymal and ejaculated sperm and the higher ICSI success rates with use of testicular sperm rather than ejaculated sperm. 


\section{FIGURE 1}

A pictorial summary of the recommendations for SDF testing and possible management in couples with high SDF
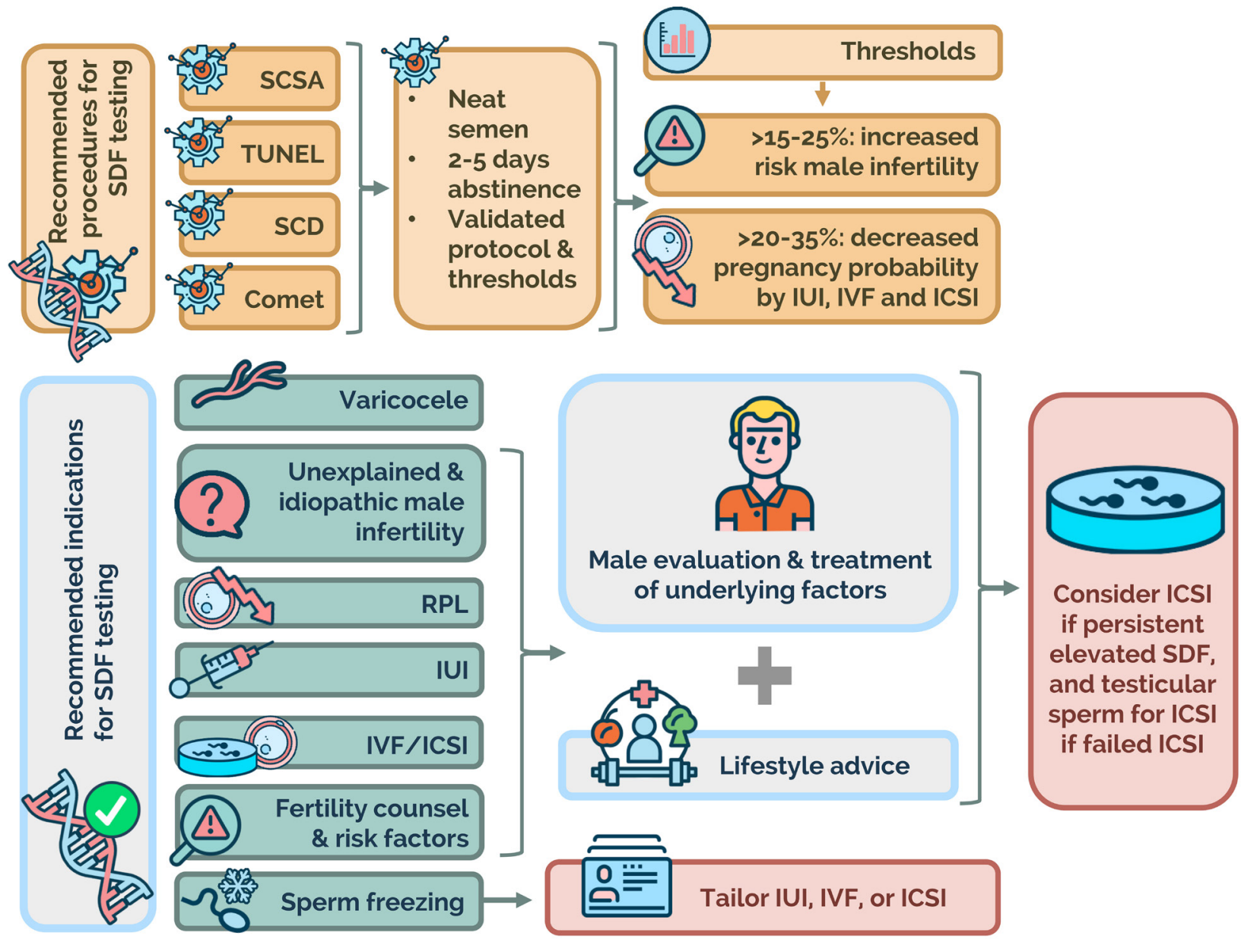

Reprinted with permission. Esteves SC, et al.[9]

IUl: intrauterine insemination; IVF: in vitro fertilization; ICSI: intracytoplasmic sperm injection; RPL: recurrent pregnancy loss.

- Risk factors: SDF testing is recommended in men with infertility risk factors (eg, tobacco smoking, obesity, metabolic syndrome, exposure to environmental or occupational toxicants, use of drugs with gonadotoxic effects, and advanced paternal age). An abnormal SDF test result may be used for counseling, reinforcing the importance of lifestyle changes and avoiding exposure to toxins, and monitoring the effect of lifestyle changes. It should also prompt a urologist's evaluation to help identify other hidden and potentially correctable conditions linked to SDF.

The SFRAG guideline was developed by reproductive urologists with clinical experience in diagnosing and treating male factor infertility and a reproductive endocrinologist with insight into the application of SDF testing in couples undergoing assisted conception. Additionally, a group of scientists pivotal in developing the main SDF assays worked together, making the interpretation of tests' results and their limitations easy to understand. For each recommendation, a strength rating based on both expert judgment and evidence levels is provided. The guideline emphasizes the central role of urologists in the evaluation of the male partner and highlights the importance of corrective measures to improve the male reproductive health overall and SDF in particular (Figure 1). Lastly, the SFRAG guideline discusses the main gaps in knowledge and provides recommendations for future research. 


\section{References}

1. Krawetz SA. Paternal contribution: new insights and future challenges. Nat Rev Genet. 2005;6:633-642.

2. Esteves SC, Santi D, Simoni M. An update on clinical and surgical interventions to reduce sperm DNA fragmentation in infertile men. Andrology. 2020;8:53-81.

3. Champroux A, Torres-Carreira J, Gharagozloo P, Drevet JR, Kocer A. Mammalian sperm nuclear organization: resiliencies and vulnerabilities. Basic Clin Androl. 2016;26:17.

4. Esteves SC, Santi D, Simoni M. An update on clinical and surgical interventions to reduce sperm DNA fragmentation in infertile men. Andrology. 2020;8:53-81.

5. Jin J, Pan C, Fei Q, Ni W, Yang X, Zhang L, et al. Effect of sperm DNA fragmentation on the clinical outcomes for in vitro fertilization and intracytoplasmic sperm injection in women with different ovarian reserves. Fertil Steril. 2015;103:910-916.
6. Aitken RJ. DNA damage in human spermatozoa; important contributor to mutagenesis in the offspring. Transl Androl Urol. 2017;6:S761-S764.

7. Esteves SC. Clinical relevance of routine semen analysis and controversies surrounding the 2010 World Health Organization criteria for semen examination. Int Braz J Urol. 2014;40:443-53.

8. Esteves SC, Agarwal A, Cho CL, Majzoub A. A strengthsweaknesses-opportunities-threats (SWOT) analysis on the clinical utility of sperm DNA fragmentation testing in specific male infertility scenarios. Transl Androl Urol.2017;6(Suppl 4):S734-S760.

9. Esteves SC, Zini A, Coward RM, Evenson DP, Gosálvez J, Lewis SEM, et al. Sperm DNA fragmentation testing: Summary evidence and clinical practice recommendations. Andrologia.2020;0ct 27:e13874. Epub ahead of print. 